\title{
Based on Agent Model and K-Core Decomposition to Analyze the Diffusion of Mass Incident in Microblog
}

\author{
Jun Pan, Huizhang Shen, and Zhong Chen \\ Antai College of Economics \& Management, Shanghai Jiao Tong University, Shanghai 200052, China \\ Correspondence should be addressed to Jun Pan; jun_a_pan@163.com
}

Received 19 April 2017; Revised 2 September 2017; Accepted 19 September 2017; Published 10 December 2017

Academic Editor: Roberto Natella

Copyright (C) 2017 Jun Pan et al. This is an open access article distributed under the Creative Commons Attribution License, which permits unrestricted use, distribution, and reproduction in any medium, provided the original work is properly cited.

\begin{abstract}
Mass incidents, which may influence the stability and security of the society in China, are getting more and more attentions not only from policy makers but also from Chinese social researchers. Catching the diffusion mechanism is believed to be critical to understand essential of these mass incidents since message dissemination plays important roles in every stage of mass incident. Recently, online social networks including Weibo (Chinese Twitter) become more and more popular in China. There are reports showing that Weibo discussion has accompanied the processes of most mass incidents happening in China in these few years. So, in this paper, we aim at introducing $K$-Core decomposition method from complex network to the analysis on how to manage the diffusion of mass incident in Weibo based on agent based model which can simulate Weibo user's actions when mass incident happens. This work can help people understand how mass incident messages spread across the network. And then, people may have better strategy to manage the diffusion of mass incidents.
\end{abstract}

\section{Introduction}

Social protest or collective protest, which is understood as the expression of intense social discontent from people, is called mass incident in China. The types of mass incidents range from tax riots to environmental protests, from land and labor disputes to ethnic clashes [1]. To a certain extent, they are normal symptoms for any society that experience profound social and economic transformation. Since such kind of mass incidents may influence the stability and security of the society in socioeconomic transition period, they get a lot of attentions not only from Chinese social researchers but also from policy makers these years.

Understanding how messages related to mass incidents diffuse is so critical for different reasons. Firstly, message exchanging in the web has accompanied the process of development in most of mass incidents. There is the common sense belief that evolution of mass incident is the procedure of its message diffusion. It implies that managing dissemination is managing mass incidents [2]. Secondly, only when people have good understanding on the mechanism of mass incident diffusion, they can construct the optimal policy response to a crisis. Last but not least, there is also the agreement among researchers that message dissemination is the root cause why mass incident can have bad society impact [3].

However, participants of mass incident are diverse, environments of mass incidents are different [4], and root causes of mass incident are complex [5]. All of these factors prevent people from getting whole picture of message diffusion when mass incident happens. Considering that agent based simulation can handle the complexity of solutions through decomposing, modeling, and organizing the interrelationships among components [6] and this method has been successfully applied in a large number of works [7], we introduce it into the discussion on mass incident diffusion. Although we only focus our research on mass incident diffusion in Weibo (Chinese Twitter) since it is one of the most popular social network platforms in China, we do believe that the analysis in this paper can be easily extended to the analysis on mass incidents diffusion in other social network platforms by similarity arguments. 


\section{An Overview of Mass Incidents and Related Works}

2.1. Mass Incidents in China. Civil disorder, also known as civil unrest or civil strife, is a broad term that is typically used by law enforcement to describe one or more forms of unrest caused by a group of people [8]. Incident of civil disorder is called mass incident in China, and mass incident is defined broadly as "planned or impromptu gathering that forms because of internal contradictions" and can include public speeches or demonstrations, physical clashes, public airings of grievances, and other group behaviors that are seen as disrupting social stability. These mass incidents regarding social issues exemplify the pains and challenges associated with China's development, and they are believed to be the expression of intense social discontent and barometers for regime stability [9]. Since mass incidents normally have bad society impact, they are now getting more and more attentions in China.

2.2. Related Works. From the perspective of research on mass incidents, the studies of mass incidents in contemporary China have become a rapid-growing industry [10]. All these jobs have well advanced our understanding of mass incidents. However, most of them are just pure "theoretical researches" from the perspective of political science, sociology, law, and management science. Without the support from underlying data, these literatures provide very limited instruction to people how to predict mass incidents, how to prevent mass incidents, and how to manage mass incidents.

From the perspective of research on message dissemination, social networks have emerged as a critical factor in the spread of information [11], marketing [12], innovation, and influence discovery. Data sets from social networks offer rich sources of evidences for studying the structure of social networks, the dynamics of individual, group behavior, global properties of information cascades [13], and identification of influential entities. Additionally, there are also global research papers with respect to microblogging service and social media in mass emergency. But, most of the available papers in this area do not touch mass incidents happening in China.

\section{Weibo, Diffusion System of Mass Incident, and Data Collection}

3.1. Weibo. Over the past years, online social networks, like Weibo (Chinese Twitter) and WeChat, have penetrated into the population in China at an accelerated pace. Among them, Weibo (Chinese Twitter) is one of the most popular microblogging social media platforms in China that enables communication among networked users, and it is experiencing a rapid increasing in its user base in China. Weibo users can broadcast an unlimited amount of messages to a group of other Weibo users (followers) who have opted to subscribe to these broadcasts. Weibo users also receive broadcasts from other users they subscribe to or are "following." Each Weibo account has a profile that contains a chosen name, location, bio, and a list for both the followers and the accounts he or she is following. Weibo had grown very rapidly over the past years in China, and it also played a pivotal role in the development of mass incidents.

3.2. Diffusion System of Mass Incident in Weibo. Most researches of online social network take the user as the node while taking static relationship (who follows who) which indicates who received whose message as the edge from the perspective of graph theory. In this paper, we keep this idea that Weibo users are mapping to the nodes of network. But edges in our research are not built directly from static relationships. Instead, we define edges of mass incident diffusion system according to the relationships of message retransmission among users. It means a directed link from node A (mapping to user A) to node B (mapping to user B) only if user B has the action (repost, reply, or reference) to the message sent by user A. Obviously, this network emerges from users' interactions instead of the passivity of the following relationship. There are two reasons behind this method. On one hand, this method can rule out silence users in the network and optimize the size of target network to be analyzed. On the other hand, it can reduce the noise raised in data collection.

In summary, if the mass incident diffusion system includes $N$ users and $M$ relationships, it can be formally defined as

$$
\begin{aligned}
G & =(V, E) \\
V(G) & =\left\{v_{i} \mid i \in\{1,2, \ldots, N\}\right\}, \quad \text { where } v_{i} \text { is mapped to user } i \\
E(G) & =\left\{e_{i} \mid i \in\{1,2, \ldots, M\}\right\},
\end{aligned}
$$

where $e_{k}=\left(v_{i}, v_{j}\right)$ means there is the message sent by user $i$ and reposted by user $j$.

3.3. Data Collection. The mass incident focused on in this paper is Jiangsu Qi dong OJI PAPER incident which happened in 2012. First Weibo message regarding this event is sent on 29 April 2012. With more and more participants involved and related messages sent through Weibo platform, diffusion of this mass incident evolved over time. After 4 
TABLE 1: Data collected from Jiangsu Qi dong OJI PAPER incident.

\begin{tabular}{lc}
\hline Description mass incident & $\begin{array}{c}\text { Jiangsu Qi dong OJI } \\
\text { PAPER incident }\end{array}$ \\
\hline Time window & 16 April 2012 27 July 2012 \\
(90 days) \\
Total \# Weibo messages & 46251 \\
Total \# participants & 28400 \\
Total \# active participants & 9222 \\
Total \# relation of repost & 57041 \\
\hline
\end{tabular}

phases and 90 days of development, message discussion reached peak on 28 May 2012 when the large scale incident happens.

Weibo maintains a suite of search APIs which allow researchers to design software to access information from Weibo in the public timeline. These searches can provide individual Weibo information including text and timestamp of messages as well as the links to the Weibo author's profile information. Using these Weibo APIs, we take three steps to collect massive data from Jiangsu Qi dong OJI PAPER incident. The first step relies on searching via key words. In this step, all messages containing key words of this mass incident are picked up if they are sent in defined time window. In Step 2, any repost or reply on the messages we get in Step 1 is collected completely. Furthermore, we have Step 3 to cover the messages which are related to this mass incident but without any popular key words. In this step, we firstly identify the participants by all messages collected in Step 1 and Step 2. After that, we go through all the messages sent by these users within the time window that Jiangsu Qi dong OJI PAPER incident happened to dig out remaining mass incident messages which are not collected well in Step 1 and Step 2.

According to this collecting method, we have sufficient data including timestamp, user profile, repost count, and reply count of messages related to this mass incident. Using these data, we can not only have user collection but also have the timeline of message dissemination in Weibo network. Moreover, these data can also be the inputs to construct whole diffusion network of this mass incident. Since the users without any followers have zero contribution to diffusion system, we choose to discard them which can help improve the efficiency of the analysis. It means that our discussion in this paper only focuses on active users. Relying on this data collection method, we build diffusion network of Jiangsu Qi dong OJI PAPER incident as shown in Table 1.

\section{Agent and Complex Network Based Model to Simulate for Mass Incident Diffusion}

Available papers have shown that social network such as Twitter, Facebook, and Weibo exhibits scale [14], complexity [15], node interaction, and emergent behavior [16] which implies that mass incident diffusion system is a complex adaptive system [17]. Traditional analytical models like differential equations cannot help people understand the evolution

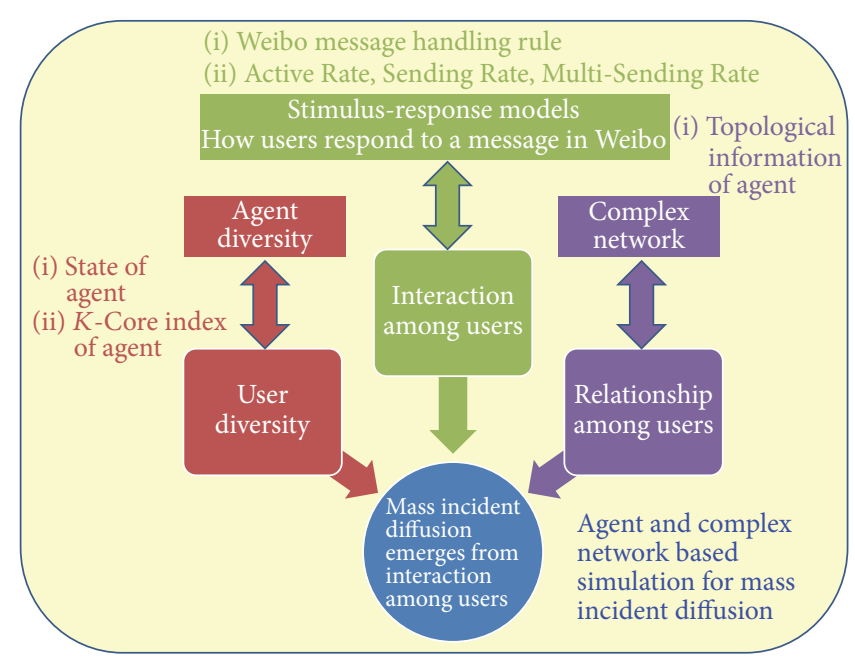

FIGURE 1: Agent and complex network based simulation for mass incident diffusion.

mechanism of such complex systems very well. Instead, agent based simulation becomes one of the popular technologies to study and model complex adaptive systems [18]. The reason behind it is agent based simulation and analysis by using a simple agent to specify single component can allow the exploration of individual level theories of behavior that can be used to examine macro level phenomenon [19]. Taking these factors into account, we choose agent based model to do our analysis in this paper.

4.1. Model Building. It is well known to all that a diffusion process in social network usually includes several essential elements:

(1) Diffusion actors representing who are involved in the part of diffusion (e.g., individuals, groups, or organizations).

(2) Reaction model of actors (e.g., what is the action from users when they get incoming messages).

(3) Diffusion medium representing the environment where the diffusion takes place, such as the connection characteristic (e.g., weak tie and strong tie) or the network structure (e.g., random network and scale free network)

To understand emergent behavior in the mass incident diffusion system, we design one agent based model to get all these elements into consideration as shown in Figure 1. In addition to two different states of agent (participant and spectator) just like the states used in SIR model (susceptible individuals, infected individuals, and recovered individuals), there are two factors which are key to this model building:

1. $K$-Core value of user is defined as one of the key properties of agent.

(2) User behavior is designed to be dynamic.

Taking these two factors into account, we can get optimized model to offer more accurate simulation result. 
4.1.1. First Key: Property of Agent and K-Core Decomposition. Expanded form of SIR is normally introduced directly into most analysis relying on agent based simulation. This method always assumes that transition rates of users are fixed, but it is not true in practice since transition rates are diversity for different users. To cover this defect and get more accurate simulation result, $K$-Core value [20] which is already recognized as a well-established method to analyze the structure of large scale graphs [21] is defined as the key property of agent in this paper. It means that we divide agents into different groups according to users' $K$-Core value. The reason why we choose $K$-Core value to identify agent group is that we find out that behavior of mass incident participant is highly correlated with $K$-Core value of this user.

Traditional researches usually rely on Poisson processes to study the mechanism of user behavior since user behavior is assumed as random in time [22]. But, as the capability of data analyzing and the methods of data collection are significantly improved, more and more empirical investigations show that many behaviors including mail [23], email [24], and other internal behaviors [25] deviate from the Poisson distribution. Instead, user behavior follows powerlaw distribution which leads to phenomenon of burst. Based on our research, this conclusion is valid as well for messages sending during the mass incident. Taking Jiangsu Qi dong OJI PAPER incident as the example, we can conclude that burst of message sending is a self-similarity property of mass incident diffusion from the perspective of $K$-Core decomposition because of the following two findings.

1. The time interval between two consecutive messages related to mass incident follows a power-law distribution. Figure 2 is the analysis for Jiangsu Qi dong OJI PAPER incident from which we can see adjusted $R$ squared is 0.855 .

2. If users are divided into different groups according to their $K$-Core values, then the time interval between two consecutive messages inside group also follows the power-law distribution. In Jiangsu Qi dong OJI PAPER incident, 21 user groups can be defined according to $\mathrm{K}$-Core decomposition. After using power-law distribution to analyze time interval between two messages sending inside every group, we summarize related adjusted $R$-squared values (Sig. = $.000)$ of all these groups in Figure 3.

Moreover, we also detect a positive correlation between the exponent of these power-law distributions and the $K$ Core value. It means that users having higher $K$-Core value normally get bigger exponent value like what is shown in Figure 4. It is because users located in inner core of diffusion network are more engaged (more frequent sending, higher transition rates) in the mass incident.

All these findings point out that $K$-Core value is highly correlated with behavior of mass incident participant. Since our target is to build a good agent model to help people get deep understand on user behavior when mass incident happens, it is believed that introducing $\mathrm{K}$-Core decomposition into model building may improve the performance of this model which may lead to more accurate simulation results.

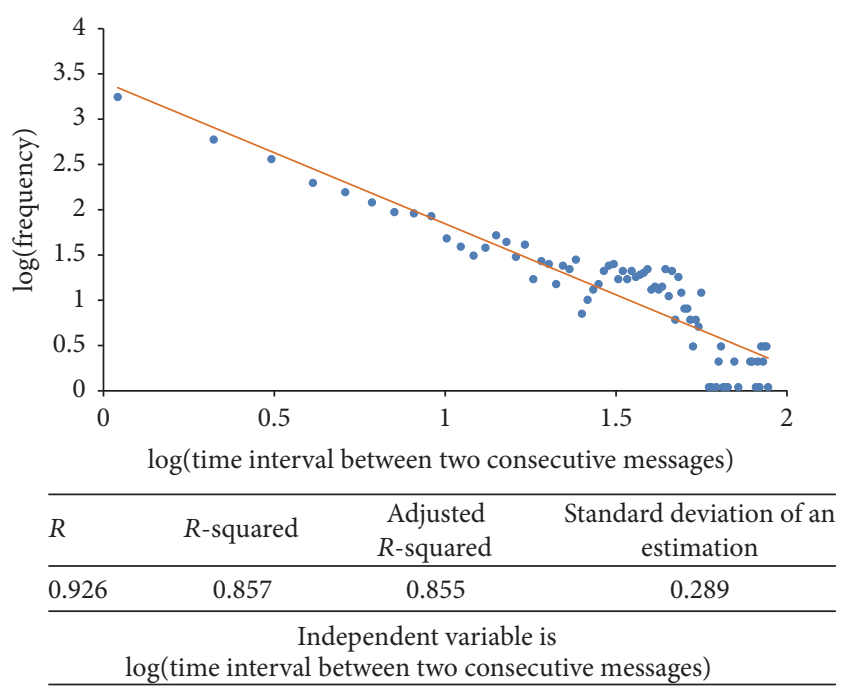

FIgURE 2: Time interval situation of mass incident.

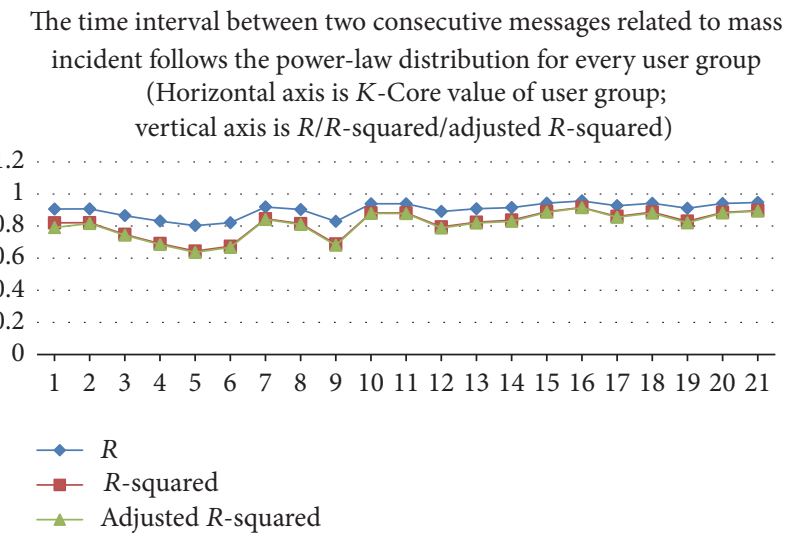

FIgure 3: Time interval of mass incident and $K$-Core decomposition (Sig. = .000). (In Jiangsu Qi dong OJI PAPER incident, 21 user groups are defined according to $K$-Core value. It shows that the time interval of messages sending is following power-law distribution inside every group.)

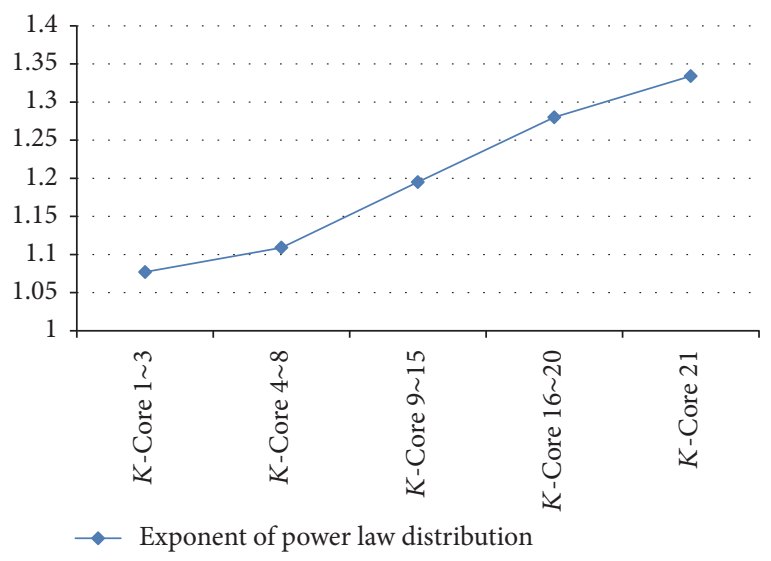

FIGURE 4: Exponent of power-law distribution for different user groups. 
4.1.2. Second Key: Interaction Rule of Agent and Dynamic Transition Rates of Users. When mass incident happens, we normally can see lots of messages related to this incident flying in Weibo. In fact, the essential of this phenomenon is that message originators create new messages and followers contribute to diffusion. It means once incident message arrives, follower makes decision whether to keep silence or take actions (repost or reply). Except the first message originator, all of other users follow process defined by stimulusresponse models [26].

Like the transition rates used in SIR model, Active Rate, Sending Rate, Multi-Sending Rate, and Immediate Action Rate are the key transition rates related to mass incident participants since they define if spectator will turn to participant, if participant will have reaction to received mass incident messages, and the detail of the reaction. However, transition rates in SIR model are fixed which are predefined before simulation. Although this handling is very simple, it may cause the accuracy problem in simulation since these rates are dynamic in practice.

In order to cover these defects and make our simulation more realistic, we investigate user behavior and come up with several key transition rates (Active Rate, Sending Rate, Multi-Sending Rate, and Immediate Action Rate) which are important to mass incident diffusion (as shown in Figure 5). Instead of predefined values, all these transition rates are designed as dynamic values according to the property and state of the user as well as the timeline.

4.2. Model Validation. In Jiangsu Qi dong OJI PAPER incident, 9222 active users are identified (as shown in Table 1), and 21 agent groups are defined according to users' $K$-Core values (as shown in Figure 3). Weibo discussion on this topic originated from the first message sent on 29 April 2012. With more participants involved day and day, this incident developed over time and we saw large scale incident finally happened on 28 July 2012. Based on the criteria if opinion leaders are involved and if public media are aware of this incident, 4 phases are identified in this case. And, matrix of

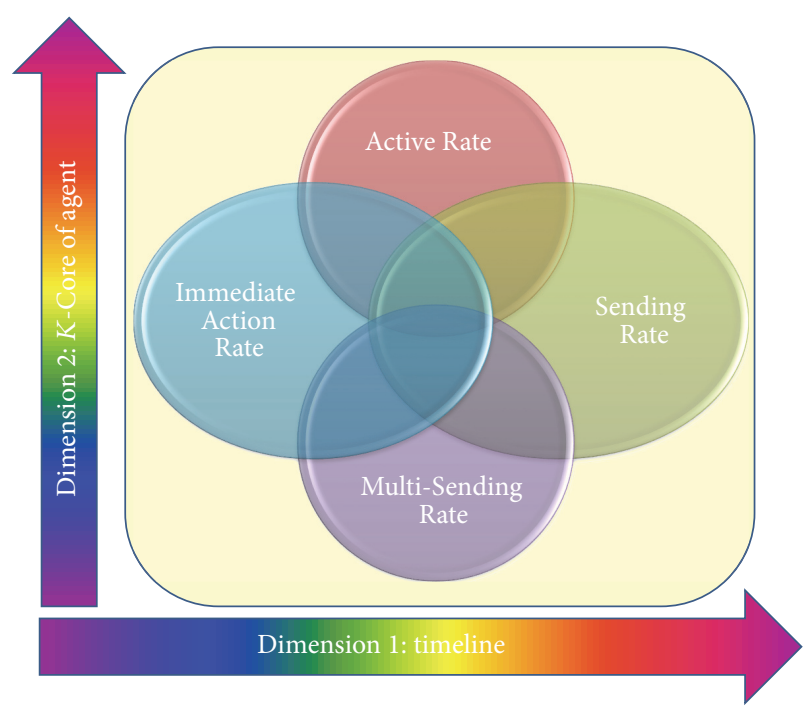

Figure 5: Dynamic user transition rates.

transition rates (Active Rate, Sending Rate, Multi-Sending Rate, and Immediate Action Rate) can be calculated based on different combinations of $K$-Core decomposition (21 agent groups) and timeline (4 phases of mass incident).

To validate this agent model by Jiangsu Qi dong OJI PAPER incident, we feed these necessary inputs as well as network structure information to this model and run 30 simulation trails. After that, we take the average of these simulations as the final simulation result, and we compare it with the observed diffusion data from two dimensions in order to get this comparison completely. One is time dimension (Figure 6) which is focusing on how accumulated receiving/sending vary in time, and another is topological dimension (Figure 7) which is focusing on what the situation of accumulated receiving/sending is among user groups defined by $K$-Core values. In detail, goodness of fit of comparison from time dimension is

$$
\begin{aligned}
R_{\text {new }}=1-\sqrt{\frac{Q}{\sum_{1}^{n} X_{i}^{2}}}= \begin{cases}0.856 & \text { (from perspective of receiving) } \\
0.929 & \text { (from perspective of sending) }\end{cases} \\
\quad\left(\text { where } Q=\sum_{1}^{n}\left(X_{i}-X_{i}^{*}\right)^{2} ; X_{i} \text { is observed data, } X_{i}^{*} \text { is simulated data }\right),
\end{aligned}
$$

while goodness of fit of comparison from $K$-Core decomposition dimension is

$$
\begin{aligned}
R_{\text {new }} & =1-\sqrt{\frac{Q}{\sum_{1}^{n} X_{i}^{2}}} \\
& = \begin{cases}0.928 & \text { (from perspective of receiving) } \\
0.873 & \text { (from perspective of sending) } .\end{cases}
\end{aligned}
$$

\section{Experimental Analysis on How to Manage Mass Incident Diffusion}

Having good agent based model to get better understand on the mechanism of mass incident diffusion is just the first step. Since the development of mass incident diffusion is nonlinear [27], the goal of our research is to provide valuable recommendation to policy makers of how to detect mass incidents timely and how to manage them effectively and 


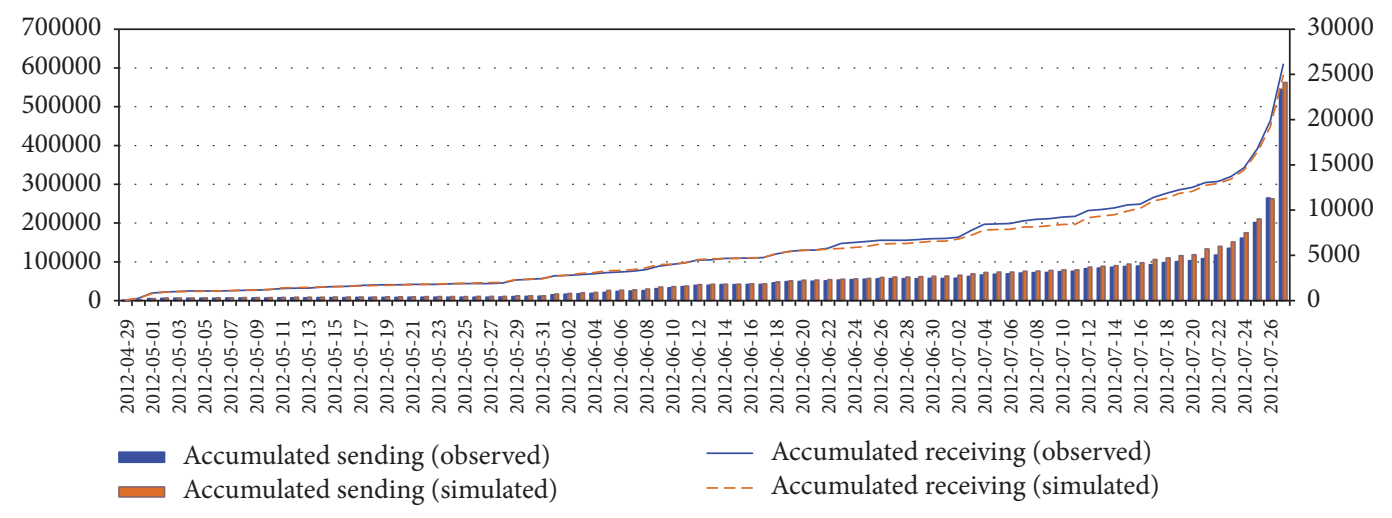

FIGURE 6: Model validation from time line.

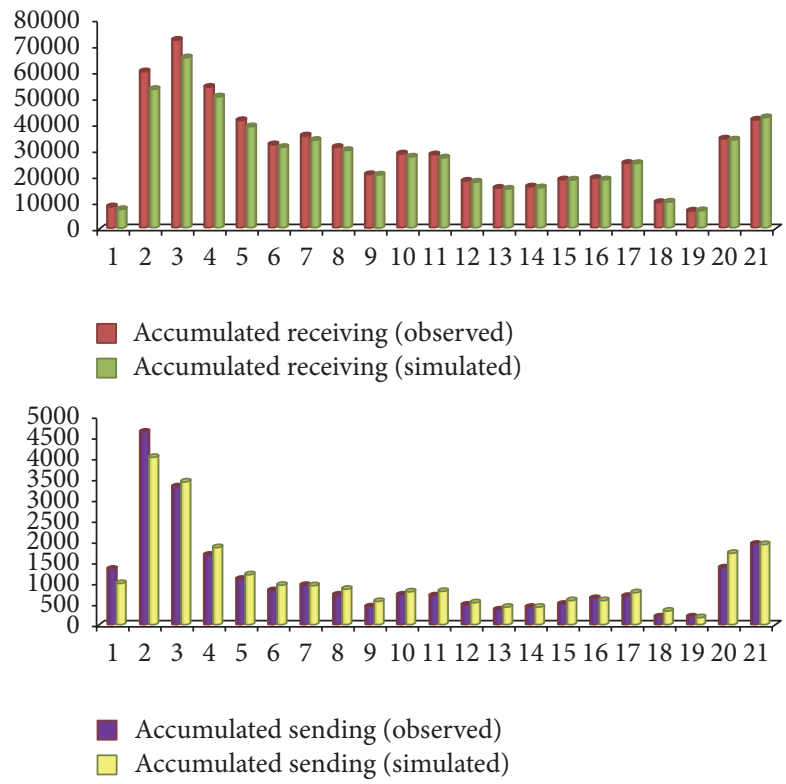

Figure 7: Model validation from $K$-Core decomposition.

efficiently. By feeding various inputs and key parameters to this agent based model, we have the possibility of showing the performance among different management strategies which may inspire people to find out better strategy to handle mass incident.

In this paper, we experiment with two kinds of "what-if" cases:

(i) What will happen to mass incident diffusion if there is positive guidance (active propaganda) in the network?

(ii) What will happen to mass incident diffusion if key users can be identified and managed during mass incident diffusion?

Taking Jiangsu Qi dong OJI PAPER incident as an example, we analyze the accumulated receiving/sending at final tick (tick 90) of this incident among different scenarios. It can help us understand what kind of management strategy may have better effectiveness.
5.1. Managing Mass Incident Diffusion by Positive Guidance (Active Propaganda). Firstly, we check the case in which policy maker can do positive guidance (propaganda) to participants via their communication channel. Once participants are influenced by this positive guidance, they may reduce their active level in this mass incident which are mapping to the translation rates (Active Rate, Sending Rate, MultiSending Rate, and Immediate Action Rate) used in our agent based model.

In order to get full picture of the impact by different strengthening level and trigger time of positive guidance, we check the accumulated receiving/sending in several detailed scenarios covering the combination of different transition rates (from 10\% decreasing to $90 \%$ decreasing) of participants and different trigger points (day 30 , day 60 , day 80 , and day 89 ) of positive guidance. We run model for 10 times and take the average outcome as a result of each scenario. And then, accumulated receiving/sending at final tick (tick 90) of each scenario along with original receiving/sending are integrated in Figures 8 and 9, respectively. In both figures, each point represents the accumulated receiving/sending under the combination of user's transition rates and trigger time of positive guidance.

From both figures, the following appears:

(1) Positive guidance is helpful to reduce the volume of messages not only from receiving perspective but also from sending perspective.

(2) Strengthening level of guidance which leads to different users' transition rates is positively correlated to the reduction level of incident messages not only from receiving perspective but also from sending perspective.

(3) The earlier positive guidance we can trigger, the more messages can be reduced not only from receiving perspective but also from sending perspective.

Based on point (2), there is no doubt that transition rates of users can be controlled to 0 when positive guidance reaches max level. And, in this case, mass incident diffusion can be totally controlled. But, since positive guidance is a cost consuming task, it may not be the right method that people like to take. Instead, it is meaningful to find out the best 


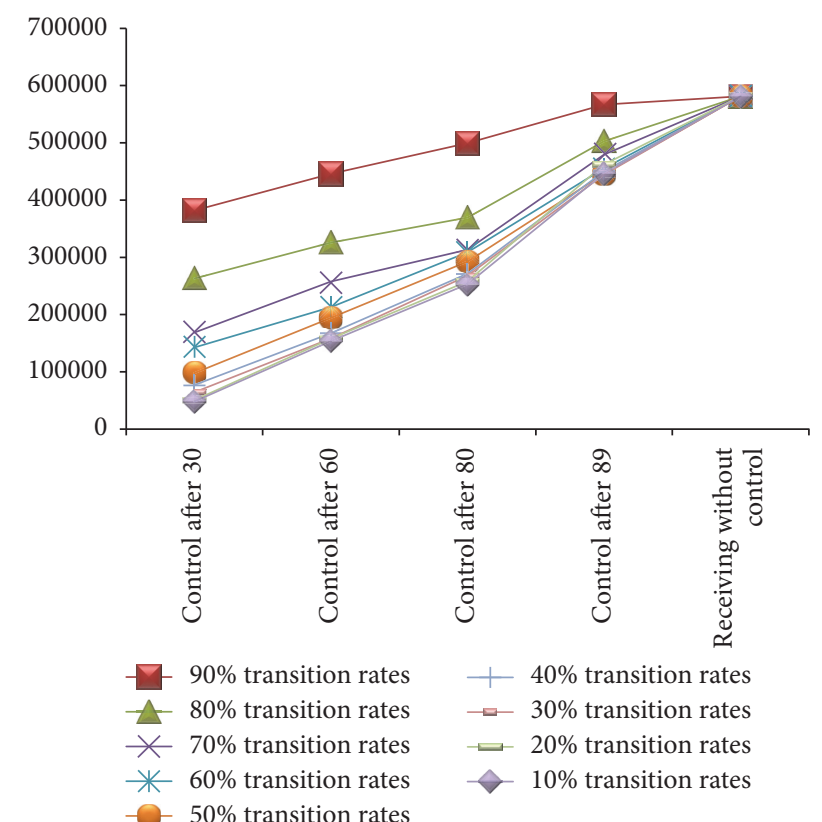

Figure 8: User transition rate, trigger point, and accumulated receiving.

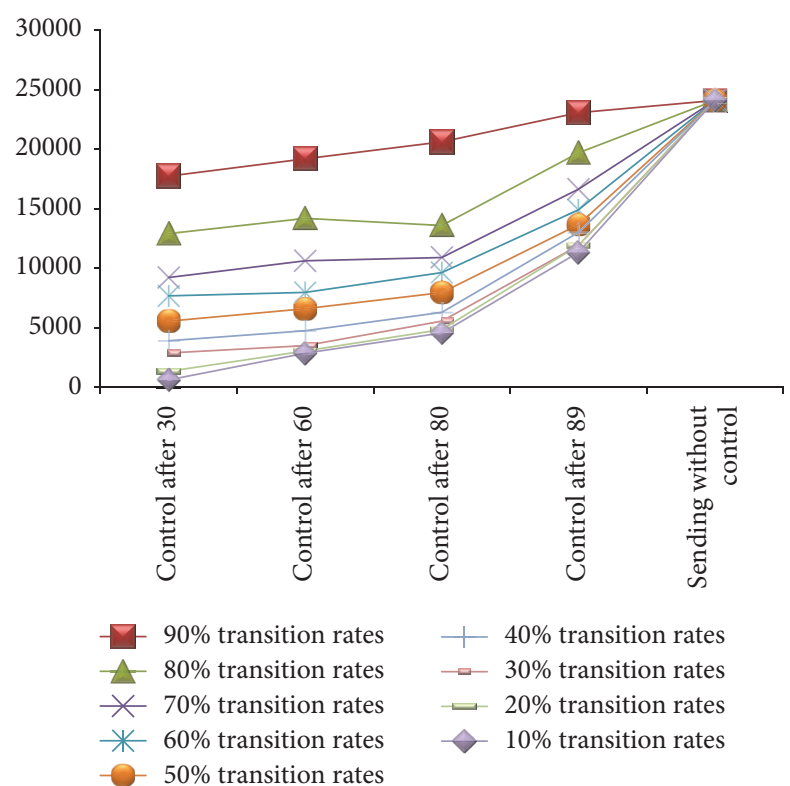

FIGURE 9: User transition rate, trigger point, and accumulated sending.

balance point between effectiveness and cost. To dig out the answer, we try the scenarios to check the changes of reduction in receiving/sending at final tick (tick 90) when users' transition rates are varied and trigger point of positive guidance is fixed. In detail, we focus on Jiangsu Qi dong OJI PAPER incident and use different colors to map different trigger points of positive guidance. And then, we measure what the reduction in final receiving/sending is for every $10 \%$ decreasing of participants' transition rates (Active Rate,

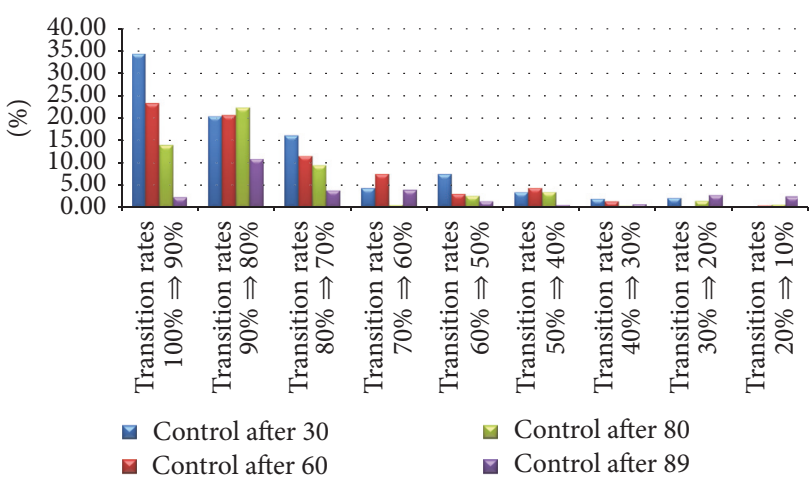

FIGURE 10: Reduction in receiving caused by user transition rates changing.

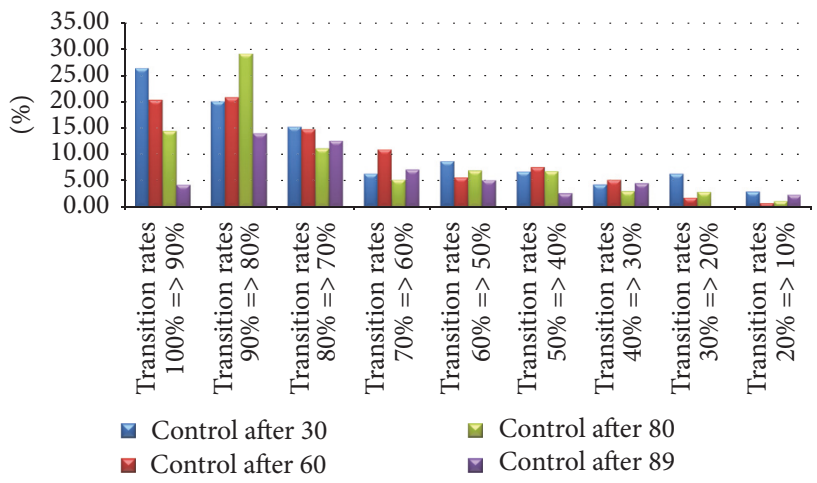

FIGURE 11: Reduction in sending caused by user transition rates changing.

Sending Rate, Multi-Sending Rate, and Immediate Action Rate) one by one. This analysis from receiving perspective is elaborated by Figure 10 while Figure 11 illustrates the situation of sending.

This analysis indicates that reduction in receiving/ sending does not vary linearly with the decreasing of users' transition rates. First three $10 \%$ decreasing of participants' transition rates $(100 \% \Rightarrow 90 \%, 90 \% \Rightarrow 80 \%, 80 \% \Rightarrow 70 \%)$ may lead to significant reduction in receiving as well as sending in the network regardless the trigger point of positive guidance. After that, further decreasing of participants' transition rates adds less and less impact to the reduction in receiving/sending. For example, blue color in Figure 10 is the scenario to analyze receiving situation when we trigger positive guidance from tick 30 . When users' transition rates are controlled from $100 \%$ to $90 \%, 90 \%$ to $80 \%$, and $80 \%$ to $70 \%$, total receiving related to this mass incident can be reduced around $35 \%, 21 \%$, and $17 \%$, respectively, compared with original receiving. But this reduction in receiving is dramatically narrowed; even users' transition rates can have further decreasing. Finally, there is only $0.5 \%$ reduction in receiving when users' transition rates have last $10 \%$ decreasing $(20 \% \Rightarrow 10 \%)$ in Jiangsu Qi dong OJI PAPER incident.

It is well known to all that it is easy to influence people and make them change a little while it is very difficult to push them to change a lot their opinion which needs huge effort 


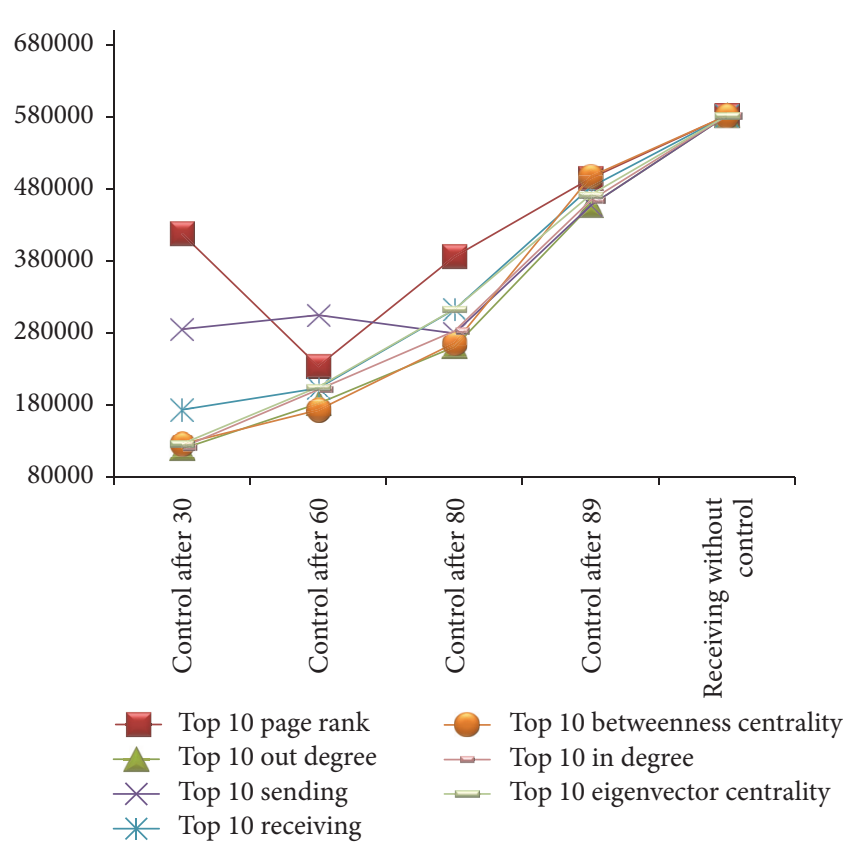

(a)

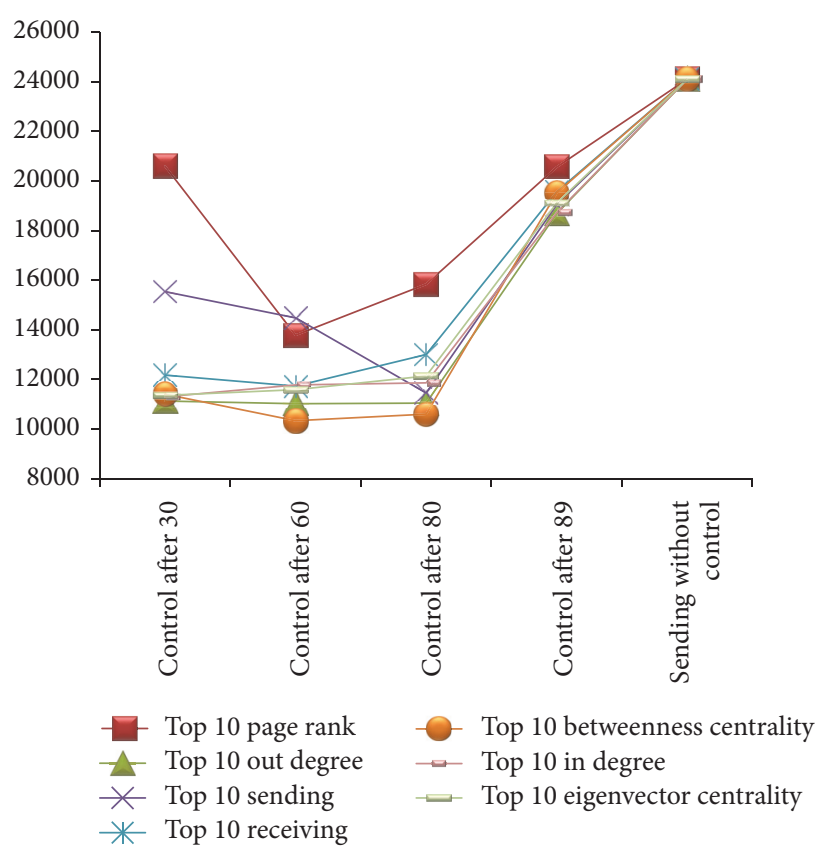

(b)

FIGURE 12: Reduction in receiving and sending by managing key users ((a) is for receiving, and (b) is for sending.).

as well as cost. However, analysis result in this section hints that the cost effective positive guidance is just focusing on how to make first $30 \%$ decreasing on users' transition rates happen. This strategy may only require few effort and cost but may lead to significant reduction in mass incident diffusion in whole network.

5.2. Managing Mass Incident Diffusion by Adding Control on Key Users. Secondly, what we want to discuss is the volume change of receiving/sending if "key users" can be managed. So, we try different scenarios to control top 10 users defined by different identification methods from the perspective of out degree, in degree, page rank, betweenness centrality, eigenvector centrality, receiving, and sending. The reasons why we choose these methods to identify the key users in the network are as follows:

(1) Degree, page rank, betweenness centrality, and eigenvector centrality are recognized as the popular index to reflect key topology property of user nodes in the area of complex network [28].

(2) Receiving volume normally reflects the exposure level of this user, and it is proved that more exposed ones are more likely to spread information [29].

(3) Receiving/sending reflecting engaging level of the user is highly correlated to user behavior when mass incident happens.

In detail, we check the accumulated receiving/sending when top 10 users are isolated from the diffusion network. Furthermore, in order to check if isolation time also impacts final result, we try 4 scenarios to cover the isolations from day 30 , day 60 , day 80 , and day 89 , respectively, for every identification method. Figure 12 summarizes the simulation results of Jiangsu Qi dong OJI PAPER incident from receiving and sending, respectively. The colors of curves indicate user identification methods we use, and the horizontal axis reflects the isolation time while the vertical axis shows the final volume of receiving in whole network.

From this figure, we can observe the following:

(1) Isolating key users can be helpful to reduce the volume of messages not only from receiving perspective but also from sending perspective.

(2) But there is the difference between this method and positive guidance. The earlier managing may not have better effectiveness to reduce final volume of receiving and sending, especially for sending. The key reason behind this phenomenon is that diffusion network of mass incident is dynamic which means that identification of key users in early phase may not be accurate.

(3) Among all these identification methods relying on out degree, in degree, page rank, betweenness centrality, eigenvector centrality, receiving, and sending, there is not one outstanding method which can have much better performance than other. The key reason is that all these methods cover either topological property of user node or behavior property of user node. One idea to improve the performance is to take both factors into account when people need to identify the key users. 
5.3. Experimental Result. So far, we have already tried two kinds of scenarios based on agent model in order to understand the effectiveness of different strategies we can take to manage mass incident diffusion.

In the first kind of scenario, we observe the changes in volume of receiving/sending when we vary users' transition rates and trigger time of positive guidance. Since we assume that positive guidance is the root cause to change users' transition rates, the essential of this analysis is to find out how to do and when to do positive guidance when mass incident happens. Based on the experimental results, we can conclude that positive guidance does make sense to manage the diffusion of mass incident. And, the earlier positive guidance can lead to better effectiveness. Moreover, although reduction in receiving/sending is positively correlated to users' transition rates, only first $30 \%$ change on users' transition rates may have significant impact to reduce the diffusion of mass incident in whole network. So, focusing on positive guidance to influence users to decrease $30 \%$ of their active level is most cost effective.

In second kind of scenario, there is the conclusion that mass incident diffusion can be reduced if key users can be identified and managed. But the difference from positive guidance is that quick action is not the key when people decide to take the approach of managing key users. Main reason is that key users in whole network are dynamic. Instead, more comprehensive method to effectively identify the real important spreaders is the key in this kind of scenario.

\section{Conclusion}

We bring some theories and methods from agent based modeling and complex network to the research on mass incident diffusion in Weibo. Firstly, based on the theory of complex system, we explain that Multi-Agent simulation is a better method to analyze the diffusion of mass incident. Based on the theory of complex network, we point out that user behavior is highly correlated with user's $K$-Core value when mass incident happens. As a result, in order to get more accurate simulation result, $K$-Core decomposition is assumed as an effective method to address the diversity of users/agents. Secondly, we brief the key points which are important to conduct and validate the agent based model used in this paper to analyze the diffusion of mass incidents in Weibo. This model is designed to understand how a mass incident might diffuse in Weibo through a large group of participants. Last but not least, we focus on this agent based model to have further discussion on how to manage the diffusion of mass incident in Weibo. We find out that the importance to positive guidance is the quick action of guidance with proper strengthening level while the key to manage key users is how to accurately identify key users.

With the growth of the use of social media, we share the view that information exchanged on social media will be faster and faster over time, and it is obvious that researches on emergence in social network will get more and more attentions. We believe that analysis and discussion in this paper can help people have better understanding on the mechanism of mass incident diffusion in Weibo. Furthermore, modeling user behavior when mass incident happens also allows people to predict the evolution of mass incident. This can be valuable inputs for policy makers to come up with better management strategy if they want to handle these issues effectively and efficiently. Although we only focus our research on Weibo in this paper, this analysis can be easily extended to the researches on mass incident diffusion in other social network platforms by similarity arguments.

\section{Conflicts of Interest}

The authors declare that they have no conflicts of interest.

\section{Acknowledgments}

(1) This research is supported by funded project (Research on the Mechanism and Risk Identification of Group Behavior, 11\&ZD174) of National Philosophy and Social Science Foundation of China. (2) This research is supported by funded project (Modeling and Experimental Study of Group Behavior in Mass Incident, 71071096) of National Natural Science Foundation of China.

\section{References}

[1] X.-D. Liu, "Depth analysis of mass incidents," Party \& Government Forum, no. 1, pp. 44-46, 2009.

[2] X.-M. Wang, "Information dissemination and management in mass incidents," Exploration and Free Views, no. 3, pp. 19-20, 2009.

[3] W. R. Dombrowsky, "Again and again: is a disaster what we call "disaster"? some conceptual notes on conceptualizing the object of disaster sociology," International Journal of Mass Emergencies and Disasters, vol. 13, no. 3, pp. 241-254, 2003.

[4] Q.-M. Dong, "The characteristics and treatment principles of mass incident," Journal of Theory Front, no. 3, pp. 7-9, 1999.

[5] J.-R. Yu, "Chinese social anger discharging events and difficulties of its management," Journal of Contemporary World and Socialism Research, no. 1, pp. 4-9, 2008.

[6] M. Niazi and A. Hussain, "Agent-based tools for modeling and simulation of self-organization in peer-to-peer, ad hoc, and other complex networks," IEEE Communications Magazine, vol. 47, no. 3, pp. 166-173, 2009.

[7] C. M. Macal and M. J. North, “Tutorial on agent-based modeling and simulation," in Simulation Conference, 2005 Proceedings of the Winter, vol. 4, p. 14, IEEE, Orlando, FL, USA, 2006.

[8] W.-J. Schurink, Victimization: Nature and Trends, Human Sciences Research Council, 1990.

[9] Chinese Public Administration Society Task Force, "Main characteristics, causes and government countermeasures of mass incidents in the transition period," Chinese Public Administration, vol. 5, pp. 6-9, 2002.

[10] Y.-F. Xu and D.-H. Liu, "The subjective game analysis for the root of mass unexpected incident," Forecasting, vol. 23, no. 6, pp. 43-45, 2004.

[11] F. Riquelme and P. González-Cantergiani, "Measuring user influence on Twitter: a survey," Information Processing \& Management, vol. 52, no. 5, pp. 949-975, 2016. 
[12] D. J. Watts and P. S. Dodds, "Influentials, networks, and public opinion formation," Journal of Consumer Research, vol. 34, no. 4, pp. 441-458, 2007.

[13] I. McCulloh and K. M. Carley, Detecting Change in Human Social Behavior Simulation, 2016.

[14] Z.-W. Tian and S. Yang, "Empirical analysis of micro-blog's information communication based on complex network theory," Library and Information Service, vol. 56, no. 8, pp. 42-46, 2012.

[15] L. D. F. Costa, F. A. Rodrigues, G. Travieso, and P. R. V. Boas, "Characterization of complex networks: a survey of measurements," Advances in Physics, vol. 56, no. 1, pp. 167-242, 2007.

[16] C. W. Johnson, "What are emergent properties and how do they affect the engineering of complex systems?" Reliability Engineering \& System Safety, vol. 91, no. 12, pp. 1475-1481, 2006.

[17] H.-Z. Shen, "Based on system theory to analyze group behaviors," Exploration and Free Views, no. 10, pp. 15-17, 2012.

[18] E. Serrano, M. Rovatsos, and J. A. Botía, "Data mining agent conversations: a qualitative approach to multiagent systems analysis," Information Sciences, vol. 230, no. 5, pp. 132-146, 2013.

[19] W. Rand and R. T. Rust, "Agent-based modeling in marketing: Guidelines for rigor," International Journal of Research in Marketing, vol. 28, no. 3, pp. 181-193, 2011.

[20] S. N. Dorogovtsev, A. V. Goltsev, and J. F. F. Mendes, "K-core organization of complex networks," Physical Review Letters, vol. 96, no. 4, Article ID 040601, 2006.

[21] C. Alves, R. Ribeiro, and R. Sanchis, "Large communities in a scale-free network," Journal of Statistical Physics, vol. 166, no. 1, pp. 137-149, 2017.

[22] M. J. G. Dominey and R. M. Hill, "Performance of approximations for compound Poisson distributed demand in the newsboy problem," International Journal of Production Economics, vol. 92, no. 2, pp. 145-155, 2004.

[23] R. D. Malmgren, D. B. Stouffer, A. S. L. O. Campanharo, and L. A. N. Amaral, "On universality in human correspondence activity," Science, vol. 325, no. 5948, pp. 1696-1700, 2009.

[24] A.-L. Barabási, "The origin of bursts and heavy tails in human dynamics," Nature, vol. 435, no. 7039, pp. 207-211, 2005.

[25] J. Yu, Y. Hu, M. Yu, and Z. Di, “Analyzing netizens' view and reply behaviors on the forum," Physica A: Statistical Mechanics and its Applications, vol. 389, no. 16, pp. 3267-3273, 2010.

[26] L. Huo, X.-M. Zhou, and H. Han, Implicit Order: Adaptability Creates Complexity, Shanghai Scientific and Technological Education, 2000.

[27] J. Pan, H.-Z. Shen, and Z. Chen, "Mass incidents and composite logistic equation," Journal of Intelligence, vol. 35, no. 5, pp. 72-78, 2016.

[28] M. Kitsak, L. K. Gallos, S. Havlin et al., "Identification of influential spreaders in complex networks," Nature Physics, vol. 6, no. 11, pp. 888-893, 2010.

[29] E. Bakshy, I. Rosenn, C. Marlow, and L. Adamic, "The role of social networks in information diffusion," in International Conference on World Wide Web ACM, vol. 8, pp. 519-528, Lyon, France, April 2012. 


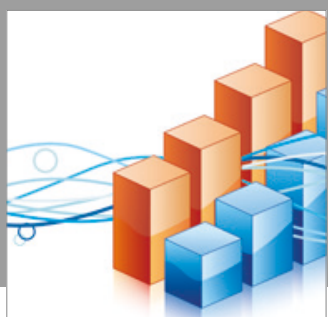

Advances in

Operations Research

vatersals

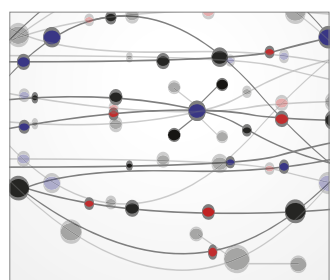

\section{The Scientific} World Journal
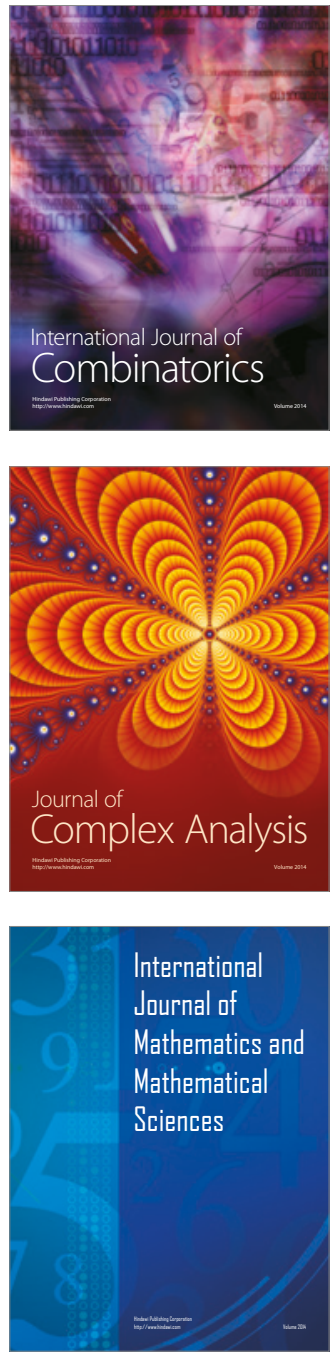
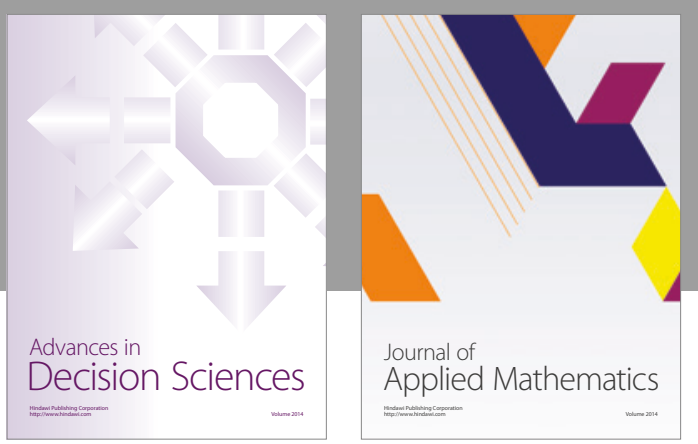

Algebra

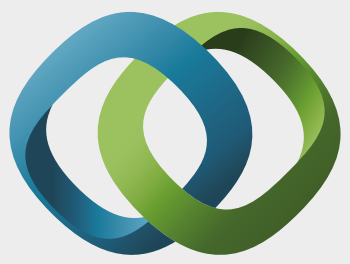

\section{Hindawi}

Submit your manuscripts at

https://www.hindawi.com
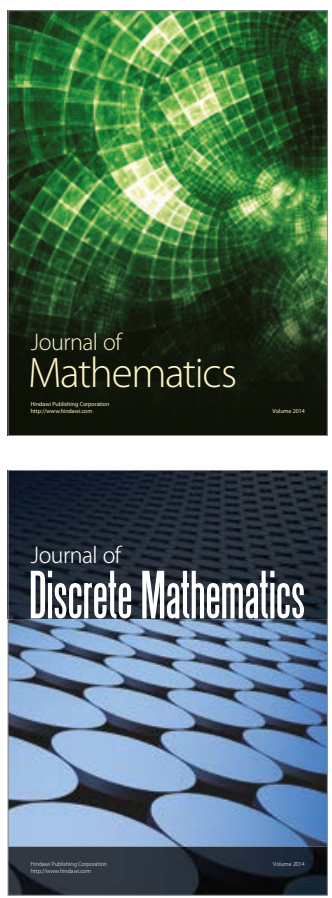

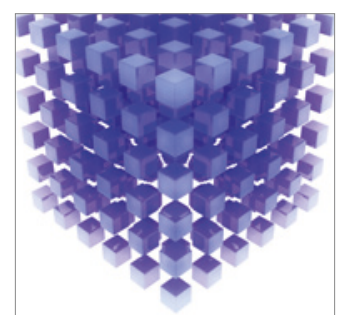

Mathematical Problems in Engineering
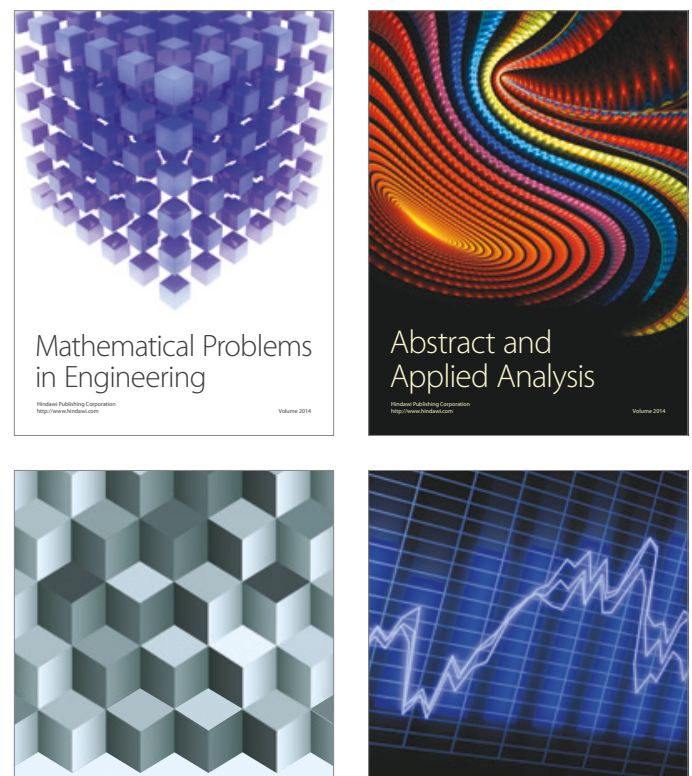

Journal of

Function Spaces

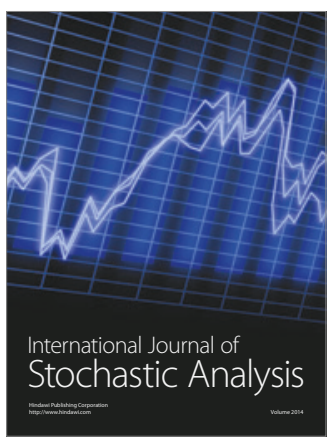

Probability and Statistics
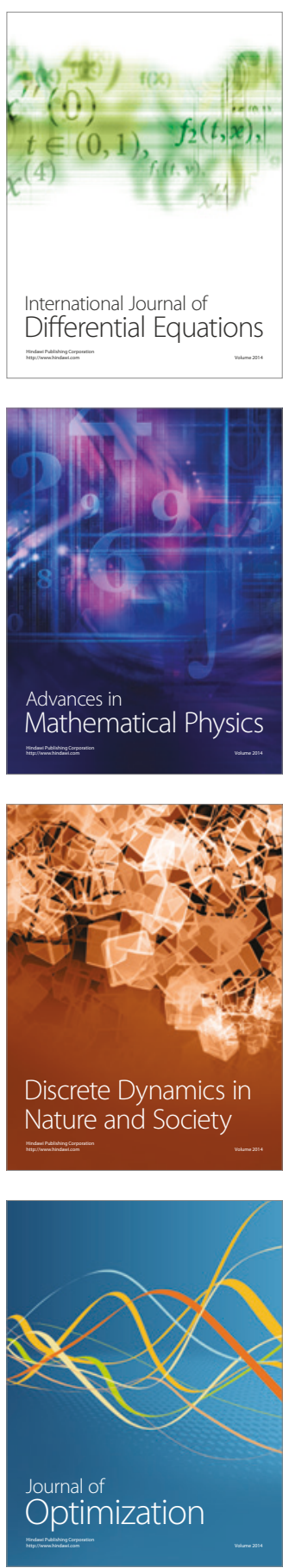